\title{
Regenerative Power Electronics Driver for Plasma Display Panel in Sustain-Mode Operation
}

\author{
Horng-Bin Hsu, Chern-Lin Chen, Senior Member, IEEE, Song-Yi Lin, and Kun-Ming Lee
}

\begin{abstract}
A regenerative power electronic circuit is proposed to drive a plasma display panel (PDP) in sustain-mode operation. This driver utilizes inductors to resonate with the equivalent intrinsic capacitance of the PDP to avoid the abrupt charging/discharging operation. The energy losses due to conventional hardswitching driving and the displacement current of the PDP are mainly recovered. Compared with prior approaches, this driving circuit has a quite simple structure and is suitable for asymmetrical operation.
\end{abstract}

Index Terms-Driver, energy recovery, plasma display panel, sustain mode.

\section{INTRODUCTION}

$\mathbf{P}$ LASMA DISPLAY panels (PDPs), invented at the University of Illinois in 1946 by Prof. Bitzer and Prof. Slottow, have advantages over conventional display technologies due to their screen size, thinness, long lifetime, and wide viewing angle. It is likely that PDPs will soon become consumer-affordable wall-hanging color TVs with large diagonal measurements. Fig. 1 shows the simplified structure of a three-electrode-type surface-discharge ac PDP. It comprises transparent $X$ and $Y$ sustain electrodes covered by a dielectric layer on the face plate, address electrodes perpendicular to the sustain electrodes on the back plate, barrier ribs for securing discharging gas space filled with discharging gas such as helium $(\mathrm{He})$ or xenon (Xe), phosphor layers over the back plate to convert the ultraviolet radiation into visible light, and a protective layer of magnesium oxide $(\mathrm{MgO})$ for protecting the dielectric layer from the discharging. By providing the phosphor layers with red, blue, and green for each pixel, a full-color plasma display can be obtained.

Like fluorescent lamps operation, the PDP utilizes the ultraviolet ray generated by gas discharge to excite phosphors encrusted on the address electrodes to emit the visible light. Applying an external electric field between the $x$ electrode and $y$ electrode will cause the filled gas to ionize. The ionized charges will be accelerated by the applied electric field. Some gas atoms

Manuscript received March 16, 1999; revised March 11, 2000. Abstract published on the Internet July 1, 2000. This paper was presented at IEEE IECON'99, San Jose, CA, November 29-December 3, 1999.

H.-B. Hsu and C.-L. Chen are with the Department of Electrical Engineering, National Taiwan University, Taipei, Taiwan 10764, R.O.C. (e-mail: f86100@ee.ntu.edu.tw; clchen@cc.ee.ntu.edu.tw).

S.-Y. Lin is with Phoenixtec, Shijr City, Taipei, Taiwan, R.O.C. (e-mail: lsy@scrd.phoenixtec.com.tw).

K.-M. Lee is with Acer Display Technology, Inc., Hsinchu, Taiwan 300, R.O.C., and also with the Department of Electrical Engineering, National Taiwan University, Taipei, Taiwan 10764, R.O.C. (e-mail: kelvinlee@adt.com.tw).

Publisher Item Identifier S 0278-0046(00)08843-2.

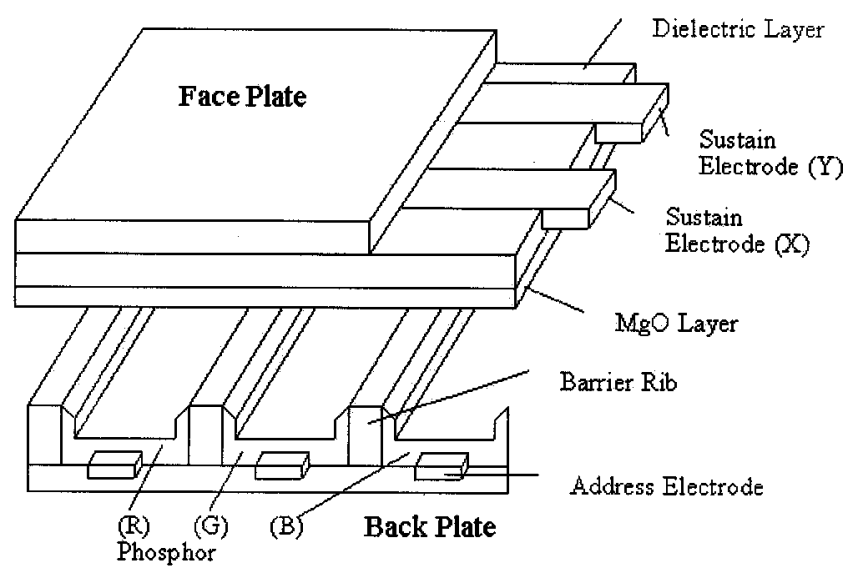

Fig. 1. Simplified structure of a three-electrode-type ac PDP.

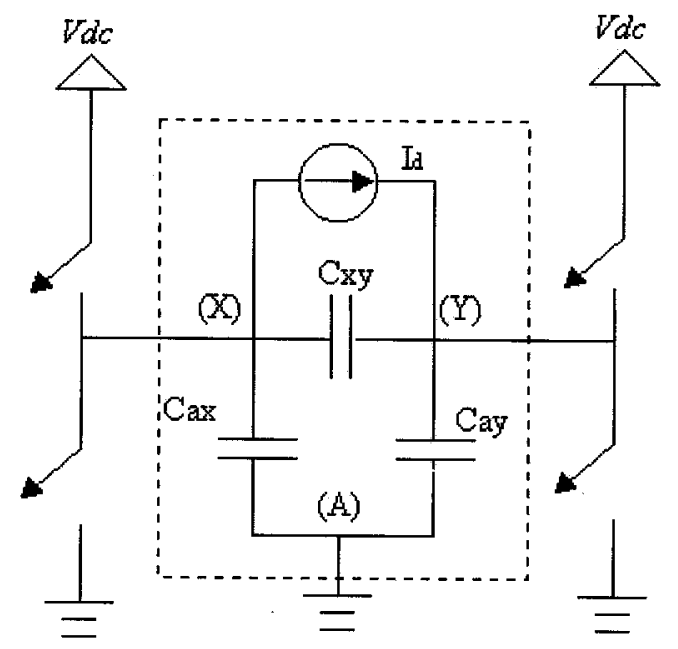

Fig. 2. Equivalent circuit of PDP with its simplest driver.

will be excited by the free electrons with enough kinetic energy. When the excited atoms return to the ground state, the ultraviolet rays are emitted and visible light is emitted after ultraviolet rays hit the phosphors.

Due to the existence of the dielectric layer, the PDP exhibits capacitive characteristic during voltage transient despite the gas discharge interval [1]. The equivalent circuit of the PDP with the simplest panel driver to generate the sustain pulse for the PDP is shown in Fig. 2 [2]. The current source $I_{d}$ is used to represent the gas discharge current. Typical values of Cxy, Cax, and Cay are about $1 \mathrm{nF}$ for an 8-inch-diagonal PDP and increase with the panel size. The values of Cxy, Cax , and Cay may be slightly affected by wall charge [1]. In this paper, however, we neglect the 


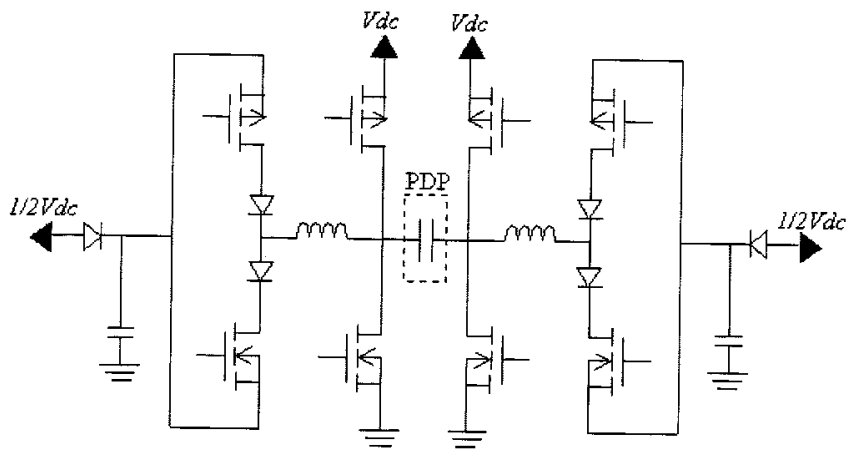

(a)

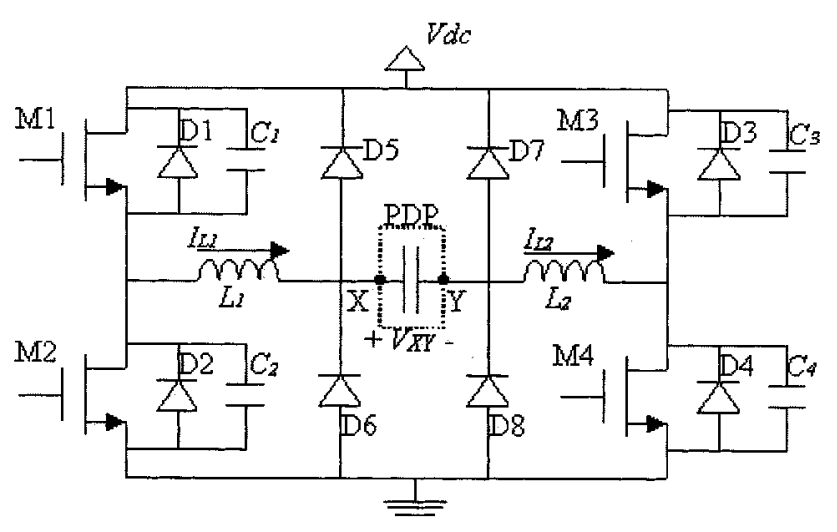

(b)

Fig. 3. Driving Circuits for PDP. (a) Prior approach. (b) Proposed driving circuit.

effect of discharge current and assume that the values of Cxy, Cax, and Cay are constant to simplify the circuit analysis. Referring to Fig. 2, the address electrodes are normally connected to ground during the sustain period and are only available during the writing or erasing operation [3], [4]. Therefore, we can replace the equivalent circuit of the PDP by a capacitor $C_{p}$. In this case, when applying a sustaining pulse to the electrodes, the energy $W$ supplied from a power source is

$$
W=C_{p}\left(V_{\mathrm{dc}}\right)^{2}
$$

where $V_{\mathrm{dc}}$ is the supplied voltage [5]. In the usual driver circuit, the energy $W$ is almost dissipated in the nonideal resistance of the wire and switching elements. For driving a large-size panel with large panel capacitance $C_{p}$ in a high-frequency high-voltage application, the energy loss will increase and better thermal design is required. Further, the surge charging/discharging current will cause a large amount of electromagnetic interference (EMI) noise, which will degrade the circuit performance.

In this paper, a regenerative driver is presented to achieve soft transient, recover capacitive energy, and reduce circuit complexity.

\section{PRIOR APPROACH}

One example of prior approaches [6]-[8] used to overcome the aforementioned problems is shown in Fig. 3(a). This circuit utilizes the resonance between the external inductor and

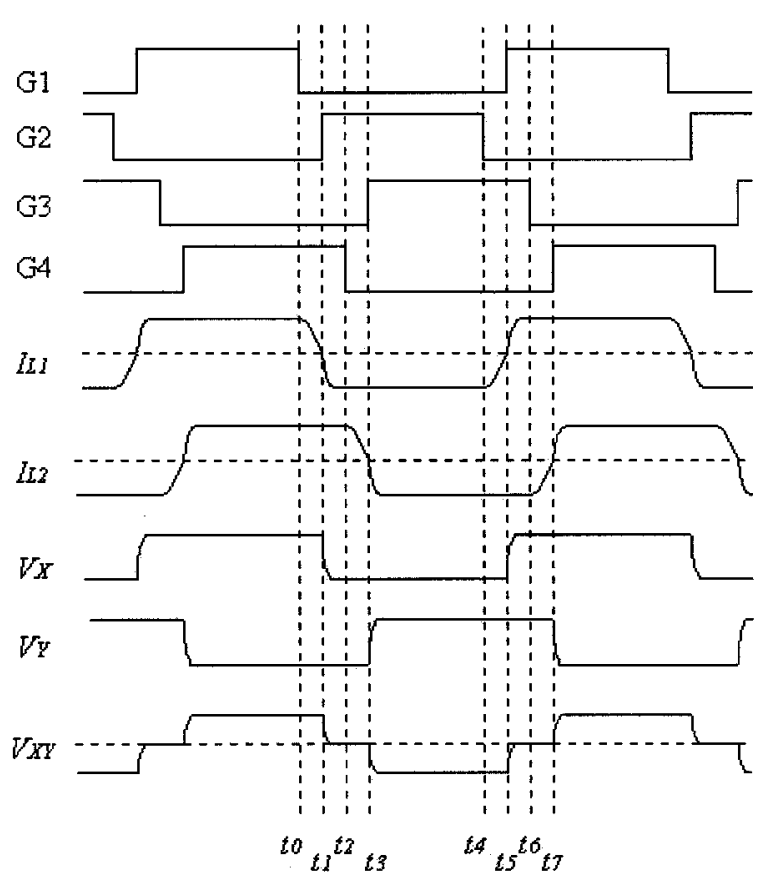

Fig. 4. Operation waveforms of the proposed driver.

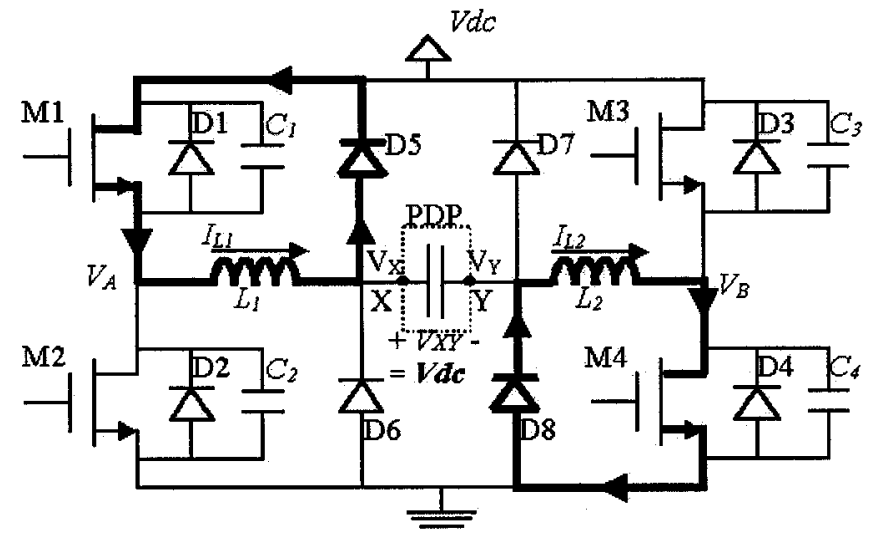

Fig. 5. Circuit operation before $t_{0}$.

the PDP to avoid abrupt charging/discharging current and, thus, saves a large amount of energy. The major disadvantage of this circuit is the circuit complexity, so that additional components and control circuits are needed. Furthermore, there will be more switching losses due to the additional switches.

\section{PRoposed Circuit}

Fig. 3(b) shows the proposed driver for the PDP. It consists of four MOSFET switches $(M 1-M 4)$ connected as a full-bridge configuration, four diodes $(D 5-D 8)$, and two inductors ( $L_{1}$ and $L_{2}$ ). Diodes $D 1-D 4$ and capacitors $C_{1}-C_{4}$ are parasitic components of the four switches. The inductors are used to resonate with the PDP. The resonant current is utilized to charge/discharge the equivalent capacitance of the PDP and switches so that the abrupt charging/discharging operation is avoided and soft transient is achieved. The diodes are used to clamp the 


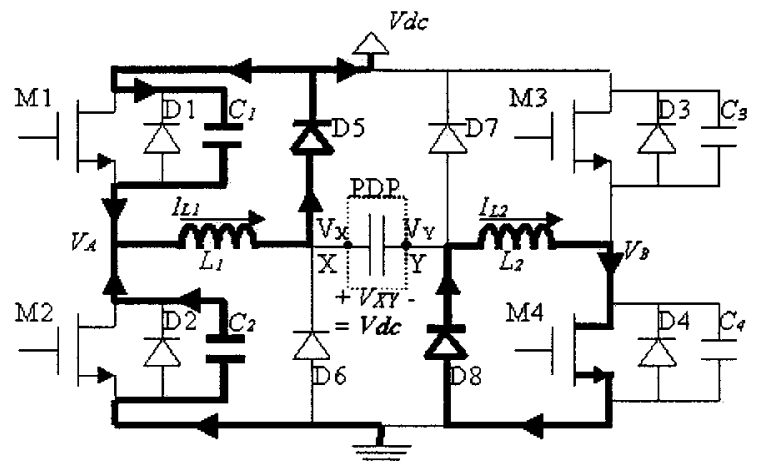

(a)

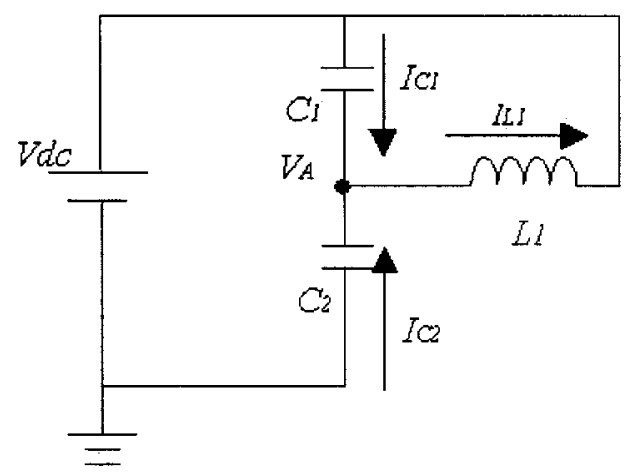

(b)

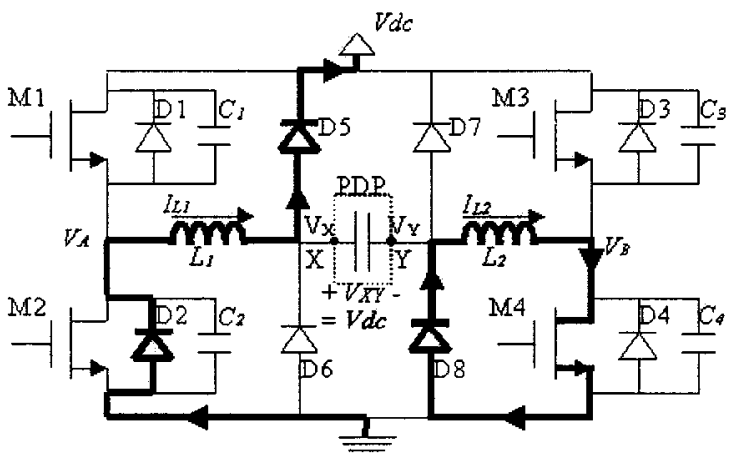

(c)

Fig. 6. Circuit operation at $t_{0}-t_{1}$. (a) Before $V_{A}$ becomes $0 \mathrm{~V}$. (b) Equivalent resonant circuit of (a). (c) After $V_{A}$ becomes $0 \mathrm{~V}$.

voltage of the PDP electrodes and conduct the extra current of the inductors. Detailed circuit operations are depicted in the next section.

\section{Circuit Operation}

Fig. 4 shows the operation waveforms of the proposed driver. G1-G4 are the respective driving signals of $M 1-M 4$. The complete cycle is divided into two halves, $t_{0}-t_{3}$ and $t_{4}-t 5$. Because the operating principles of the two half cycles are symmetric, we only explain that of the first half cycle.

\section{A. Stage 0 (Before $t_{0}$ )}

As shown in Fig. 5, $M 1, M 4, D 5$, and $D 8$ are conductive before $t_{0}$. The $X$-side voltage of the $\operatorname{PDP}\left(V_{X}\right)$ is equal to the dc-bus voltage $\left(V_{\mathrm{dc}}\right)$ and the $Y$-side voltage of the PDP $\left(V_{Y}\right)$ is equal to zero. Therefore, the voltage across the PDP $\left(V_{X Y}\right)$ is equal to $V_{\mathrm{dc}}$. At the same time, the voltages across $L_{1}$ and $L_{2}$ are zero so that the inductor current will keep constant. The inductor current of $L_{1}\left(I_{L 1}\right)$ circulates through $D 5$ and $M 1$, and the inductor current of $L_{2}\left(I_{L 2}\right)$ circulates through $M 4$ and $D 8$.

\section{B. Stage $1\left(t_{0}-t_{1}\right)$}

At $t_{0}, M 1$ is turned off and $I_{L 1}$ starts to charge $C_{1}$ and discharge $C_{2}$ at the same time, as indicated in Fig. 6(a). Fig. 6(b) shows the equivalent resonant circuit. In general, we suppose that $C_{1}$ and $C_{2}$ are equal. With initial condition $V_{A}\left(t_{0}\right)=V_{\mathrm{dc}}$, we have

$$
\begin{gathered}
V_{A}(t)=V_{\mathrm{dc}}-A_{1} \sin \left(\frac{t-t_{0}}{\sqrt{2 L_{1} C_{1}}}\right) \\
I_{L 1}(t)=\frac{A_{1} \sqrt{2 C_{1}}}{\sqrt{L_{1}}} \cos \left(\frac{t-t_{0}}{\sqrt{2 L_{1} C_{1}}}\right)
\end{gathered}
$$

where $A_{1}$ is a constant and will be discussed later.

After $V_{A}$ drops to $0 \mathrm{~V}, D 2$ becomes conductive and $I_{L 1}$ will flow through $D 2$ and $D 5$, as indicated in Fig. 6(c). Now, the voltage across $L_{1}$ is $-V_{\mathrm{dc}}$ so that $I_{L 1}$ begins to decrease linearly with slope $V_{\mathrm{dc}} / L_{1}$ and the energy stored in $L_{1}$ is recovered back to the dc bus. Then, $D 5$ and $D 2$ will be turned off naturally without the problem of reverse recovery when $I_{L 1}$ becomes zero.

\section{Stage $2\left(t_{1}-t_{2}\right)$}

After $I_{L 1}$ decreases to zero, $M 2$ is turned on with zero-voltage transition (ZVT) at $t_{1}$. Now, $V_{X Y}$ is equal to $V_{\mathrm{dc}}$ and $C_{p}$ will resonate with $L_{1}$ through $M 2$ and $D 8$, as depicted in Fig. 7(a). The equivalent resonant circuit is shown in Fig. 7(b). With initial conditions $V_{X Y}\left(t_{1}\right)=V_{\mathrm{dc}}$ and $I_{L 1}\left(t_{1}\right)=0$, we can derive the following equations:

$$
\begin{aligned}
V_{X Y}(t) & =V_{\mathrm{dc}} \cos \left[w\left(t-t_{1}\right)\right] \\
I_{L 1}(t) & =-\frac{V_{\mathrm{dc}} \sqrt{C_{p}}}{\sqrt{L_{1}}} \sin \left[w\left(t-t_{1}\right)\right]
\end{aligned}
$$




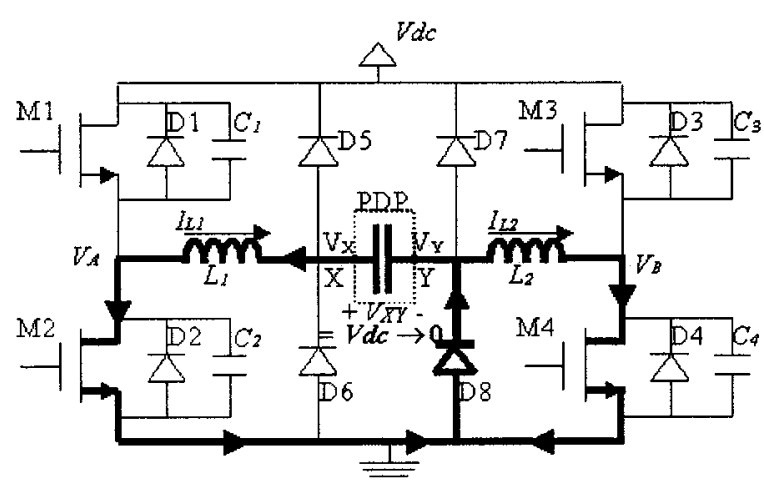

(a)

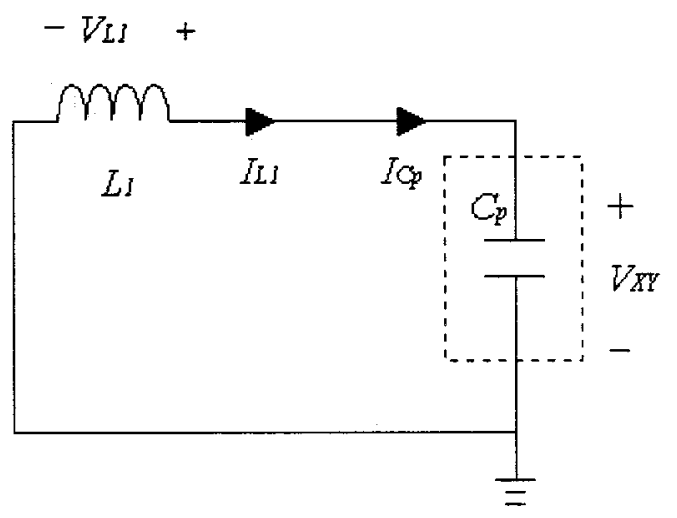

(b)

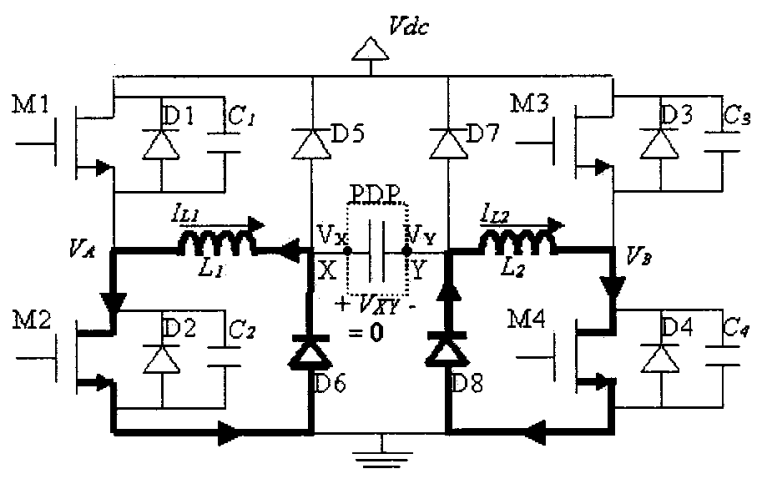

(c)

Fig. 7. Circuit operation at $t_{1}-t_{2}$. (a) Before $V_{X Y}$ becomes $0 \mathrm{~V}$. (b) Equivalent resonant circuit of (a). (c) After $V_{X Y}$ becomes $0 \mathrm{~V}$.

where

$$
w=\frac{1}{\sqrt{C_{p} L_{1}}} .
$$

When $V_{X Y}$ decreases to $0 \mathrm{~V}, D 6$ becomes conductive and $V_{x}$ is clamped at $0 \mathrm{~V}$, as indicated in Fig. 7(c). The voltage across $L_{1}$ is $0 \mathrm{~V}$ here, and $I_{L 1}$ will keep constant.

\section{Stage $3\left(t_{2}-t_{3}\right)$}

At $t_{2}, M 4$ is turned off and $I_{L 2}$ starts to charge $C_{4}$ and discharge $C_{3}$ at the same time, as indicated in Fig. 8(a). The equivalent resonant circuit is shown in Fig. 8(b). With initial condition $V_{B}\left(t-t_{2}\right)=0$, we have

$$
\begin{aligned}
& V_{B}(t)=A_{2} \sin \left(\frac{t-t_{2}}{\sqrt{2 L_{2} C_{3}}}\right) \\
& I_{L 2}(t)=\frac{A_{2} \sqrt{2 C_{3}}}{\sqrt{L_{2}}} \cos \left(\frac{t-t_{2}}{\sqrt{2 L_{2} C_{3}}}\right)
\end{aligned}
$$

where $A_{2}$ is a constant and will be discussed later.

After the voltage of $C_{4}$ rises to $V_{\mathrm{dc}}, D 3$ becomes conductive and $I_{L 2}$ will flow through $D 3$ and $D 8$, as indicated in Fig. 8(c). The energy stored in $L_{2}$ is recovered back to the dc bus. Then, $D 3$ and $D 8$ are turned off naturally without the problem of reverse recovery when $I_{L 2}$ becomes zero.

\section{E. Stage $4\left(t_{3}-t_{4}\right)$}

After $I_{L 2}$ decreases to zero, $M 3$ is turned on with ZVT at $t_{3}$ and $L_{2}$ starts to resonate with $C_{p}$, as depicted in Fig. 9(a).
Because the energy for charging the PDP is equal to that of discharging the PDP, $I_{L 2}$ will not become larger than $I_{L 1}$ if $L_{2}$ is not smaller than $L_{1}$. Therefore, $D 6$ keeps conductive and $V_{X}$ remains zero. The equivalent resonant circuit is shown in Fig. 9(b). With initial conditions $V_{X Y}\left(t_{3}\right)=0$ and $I_{L 2}\left(t_{3}\right)=0$, we can derive following equations:

$$
\begin{aligned}
V_{X Y}(t) & =-V_{\mathrm{dc}}+V_{\mathrm{dc}} \cos \left[w^{\prime}\left(t-t_{3}\right)\right] \\
I_{L 2}(t) & =-\frac{V_{\mathrm{dc}} \sqrt{C_{p}}}{\sqrt{L_{2}}} \sin \left[w^{\prime}\left(t-t_{3}\right)\right]
\end{aligned}
$$

where $w^{\prime}=\left(1 / \sqrt{C_{p} L_{2}}\right)$.

When $V_{X Y}$ decreases to $-V_{\mathrm{dc}}, D 7$ becomes conductive and $V_{Y}$ is clamped at $V_{\mathrm{dc}}$, as indicated in Fig. 9(c). Now, the voltage across $L_{2}$ is zero and the inductor current of $L_{2}$ will keep constant.

Circuit operations of $t_{4}-t_{7}$ are analogous with that of $t_{0}-t_{4}$.

\section{DESIGN CONSIDERATION}

In practical implementation, the values of $L_{1}$ and $L_{2}$ are equal. Therefore, (3-b) and (5-b) can be rewritten as

$$
\begin{aligned}
& I_{L 1}(t)=-\frac{V_{\mathrm{cc}} \sqrt{C_{p}}}{\sqrt{L_{1}}} \sin \left[w\left(t-t_{1}\right)\right]=-I_{0} \sin \left[w_{0}\left(t-t_{1}\right)\right] \\
& I_{L 2}(t)=-\frac{V_{\mathrm{dc}} \sqrt{C_{p}}}{\sqrt{L_{2}}} \sin \left[{ }^{\prime} w\left(t-t_{3}\right)\right]=-I_{0} \sin \left[w_{0}\left(t-t_{3}\right)\right]
\end{aligned}
$$




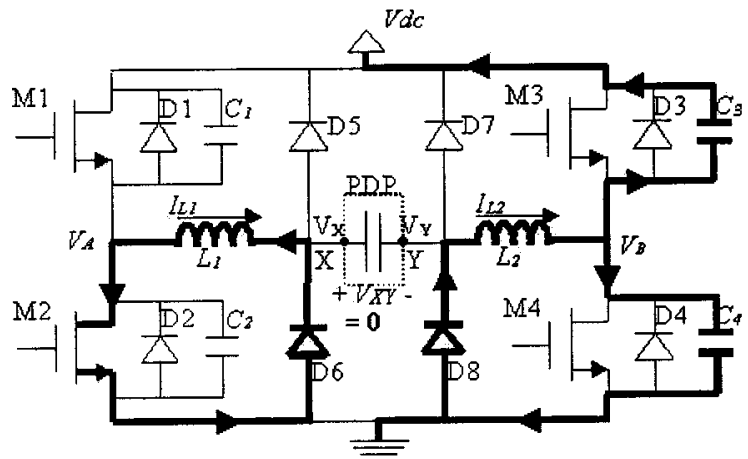

(a)

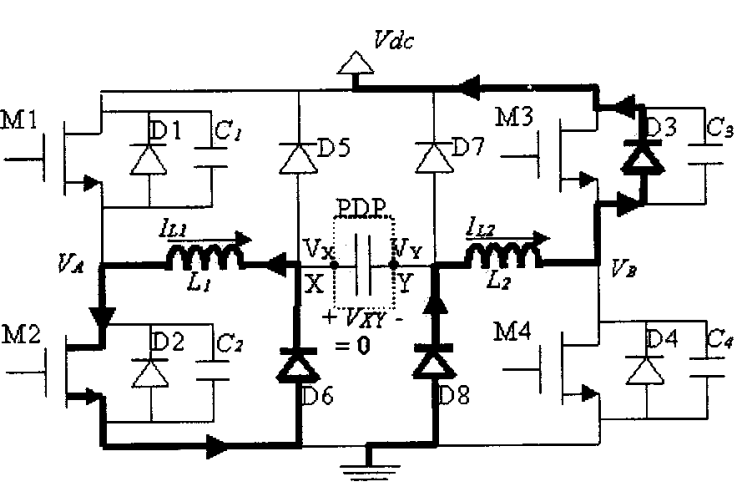

(c)

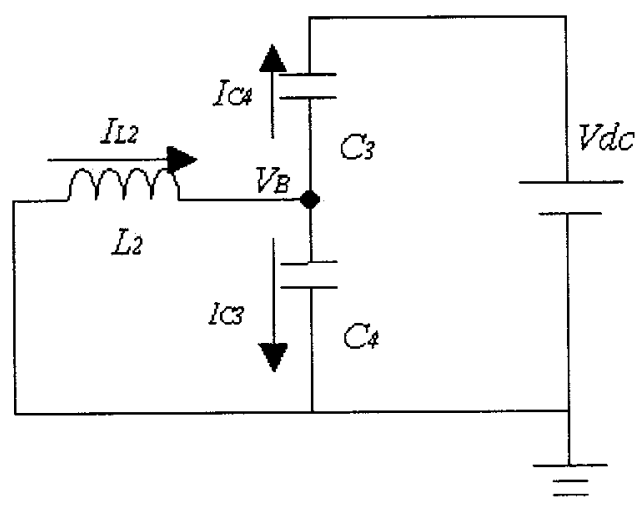

(b)

Fig. 8. Circuit operation at $t_{2}-t_{3}$. (a) Before $V_{B}$ becomes $V_{\mathrm{cc}}$. (b) Equivalent resonant circuit of (a). (c) After $V_{B}$ becomes $V_{\mathrm{dc}}$.

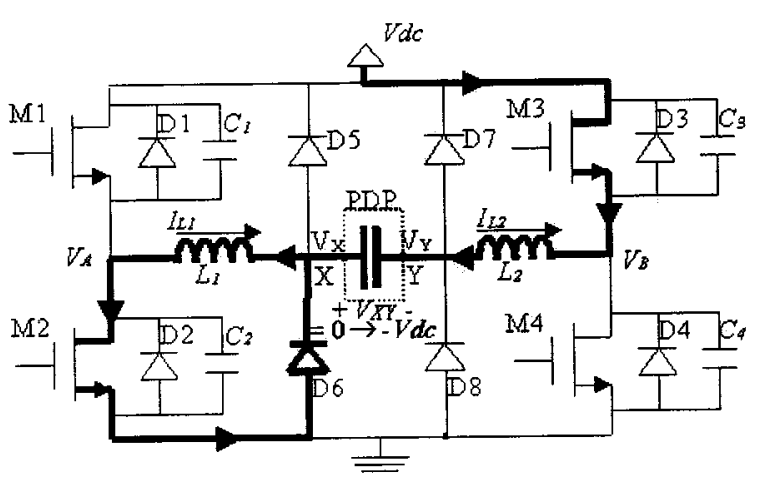

(a)

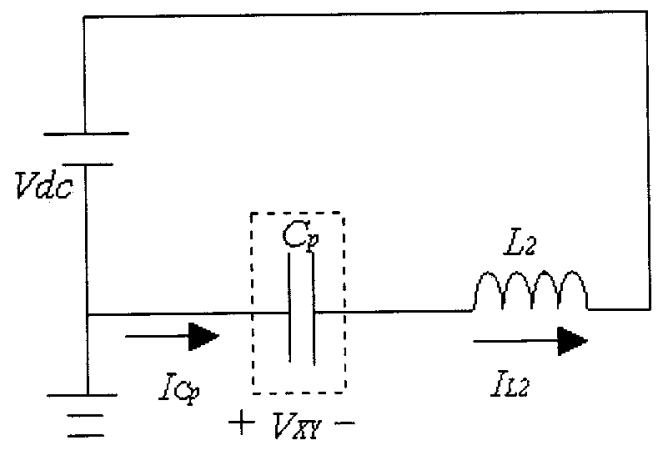

(b)

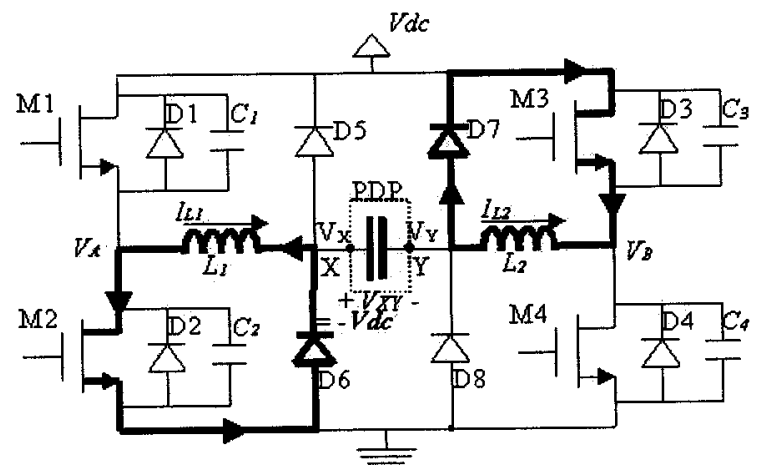

(c)

Fig. 9. Circuit operation at $t_{3}-t_{4}$. (a) Before $V_{X Y}$ becomes $-V_{\mathrm{cc}}$. (b) Equivalent resonant circuit of (a). (c) After $V_{X Y}$ becomes $-V_{\mathrm{clc}}$. 


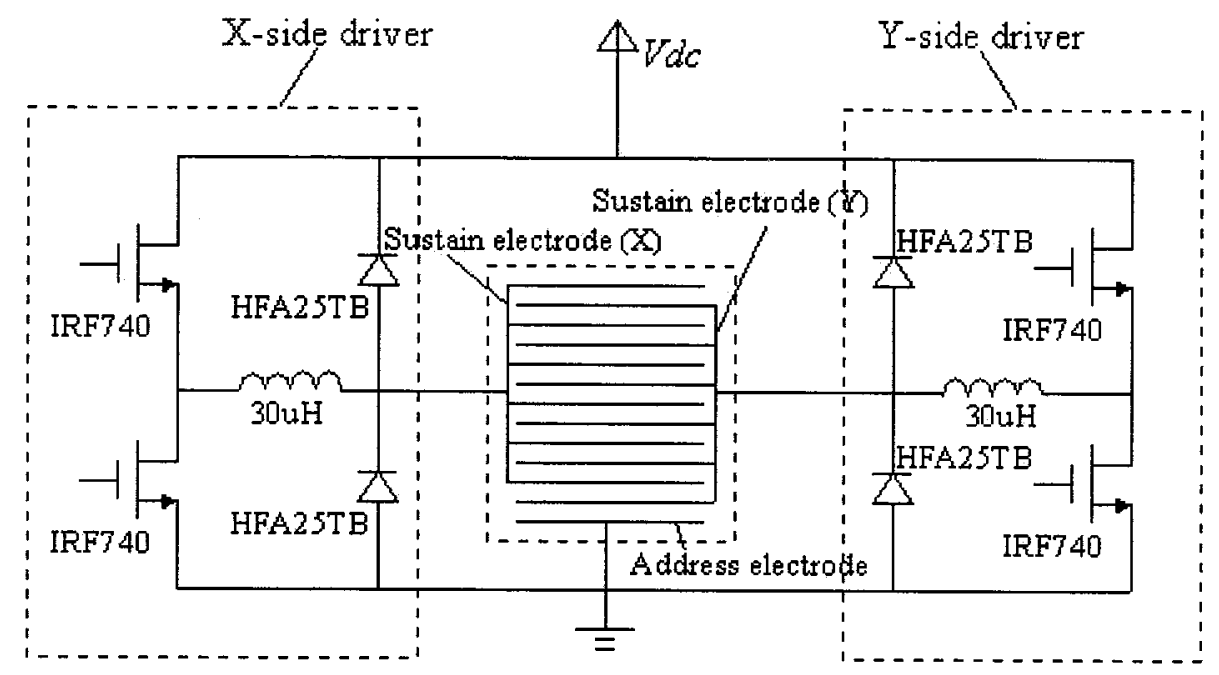

Fig. 10. Experimental circuit and associated parameters.

where

and

$$
I_{0}=\frac{V_{\mathrm{dc}} \sqrt{C_{p}}}{\sqrt{L_{1}}}=\frac{V_{\mathrm{dc}} \sqrt{C_{p}}}{\sqrt{L_{2}}}
$$

$$
w_{0}=\frac{1}{\sqrt{C_{p} L_{1}}}=\frac{1}{\sqrt{C_{p} L_{2}}} .
$$

From these equations, we know that the initial conditions of $I_{L 1}$ at stage 1 and $I_{L 2}$ at stage 2 are equal to $I_{0}$. Comparing with (2-b) and (4-b), we get

$$
A_{1}=\frac{V_{\mathrm{dc}} \sqrt{C_{p}}}{\sqrt{2 C_{1}}}=\frac{V_{\mathrm{dc}} \sqrt{C_{p}}}{\sqrt{2 C_{3}}}=A_{2} .
$$

Because $C_{p}$ is typically much larger than MOSFET parasitic capacitance, $C_{1}-C_{4}, A_{1}$ and $A_{2}$ are much larger than $V_{\mathrm{dc}}$. To achieve ZVT in this driving circuit, each switch must be turned on after its parasitic capacitor has been fully discharged. Therefore, from (2-b) and (3-b), we can get

$$
\text { dead time }>\pi \sqrt{\frac{L_{1} C_{1}}{2}} .
$$

There is no rigorous way to determine the value of the inductors, however, make sure that it is large enough to avoid abrupt charging/discharging current but will not reduce the response rate.

The switching frequency is determined by the PDP. The luminance of the PDP increases with the frequency. In practical application, the PDP usually works from 80 to $150 \mathrm{kHz}$.

\section{EXPERIMENTAL RESULTS}

Fig. 10 shows the power stage of the experimental circuit with its associated parameters. The plasma display panel under test is 8 in diagonally. Sustain electrodes $(X)$ and $(Y)$ are connected to $X$-side and $Y$-side drivers, respectively. Address electrodes are shorted together and connected to ground reference.

The control signals of $M 1-M 4$ are obtained by using a fullbridge phase-shift pulsewidth modulation (PWM) control IC,

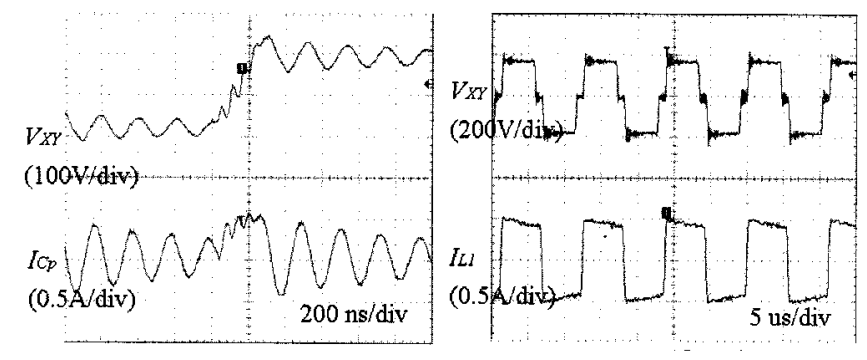

(a)

(b)

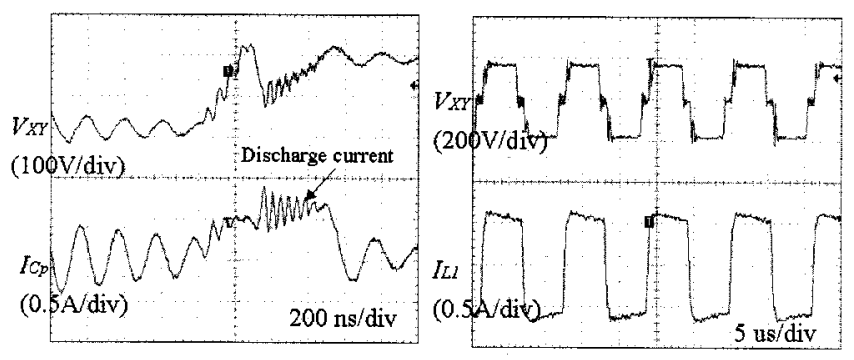

(c)

(d)

Fig. 11. Experimental waveforms of the proposed circuit. (a) Voltage across PDP and current of PDP (no light emitted). (b) Voltage across PDP and inductor current of $L_{1}$ (no light emitted). (c) Voltage across PDP and current of PDP (light emitted). (d) Voltage across PDP and inductor current of $L_{1}$ (light emitted).

UC3875. The switching frequency is $90 \mathrm{kHz}$, which satisfies the practical application of the PDP. The phase shift angle is about $150^{\circ}$ to make sure that the gas discharge of the PDP has finished.

Fig. 11 shows the experimental waveforms of the proposed circuit. In Fig. 11(a), we can find that soft current transient is derived by using the proposed circuit. A quite ideal waveform of inductor current $I_{L 1}$ is also shown in Fig. 11(b). For the case shown in Fig. 11(c) and (d), there is light emitted and apparent discharging current appears in Fig. 11(c). The waveforms of $V_{X Y}$ and $I_{L 1}$ are slightly affected by the gas discharge current. 


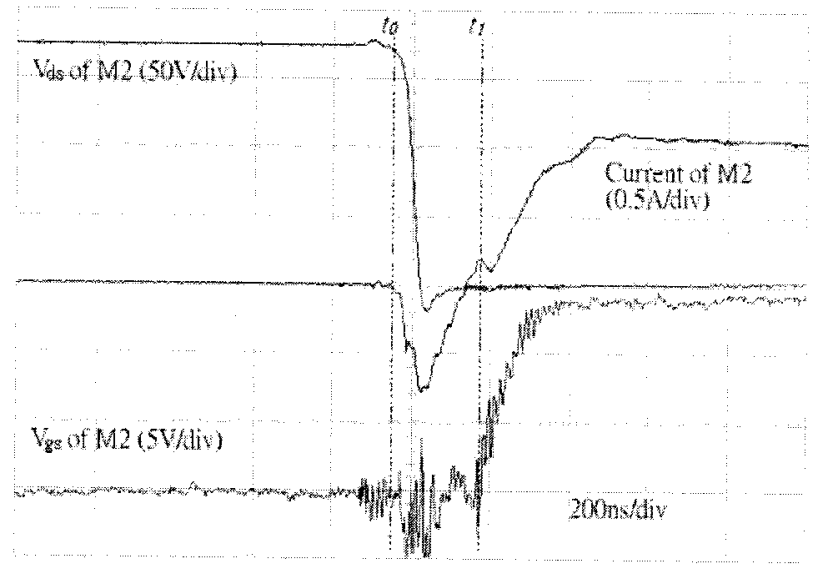

(a)

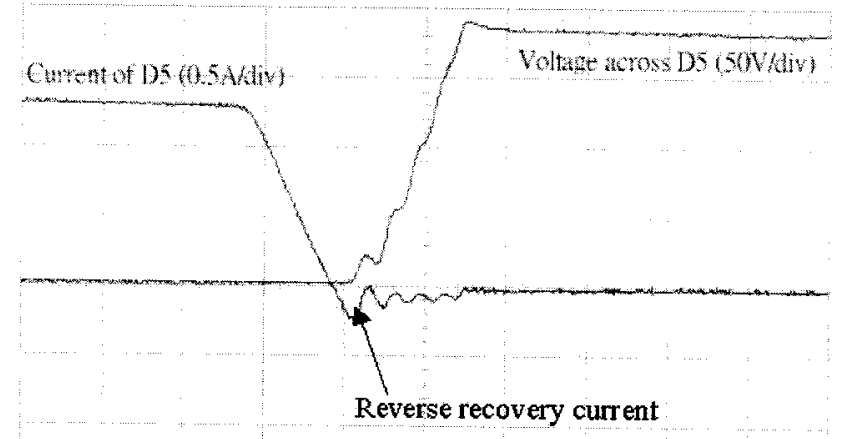

$200 \mathrm{nsdl}$

(b)

Fig. 12. Soft transition of MOSFETs and diodes. (a) Turn-on transient of $M 2$. (b) Turn-off transient of $D 5$.

The waveforms of Fig. 12 show that soft transition of the switches and the diodes is achieved in the proposed circuit. In Fig. 12(a), we could find that the inductor current starts to flow through $M 2$ to discharge $C 2$ at $t_{0}$ so that the $V_{\mathrm{ds}}$ of $M 2$ decreases. After the $V_{\mathrm{ds}}$ becomes $0 \mathrm{~V}, M 2$ is turned on at $t_{1}$ with ZVT. The turn-off transient of $D 5$ is shown in Fig. 12(b). The reverse recovery current of $D 5$ is quite small we could know that $D 5$ is turned off naturally. Because the $X$-side and $Y$-side drivers are symmetric, we conclude that all the switches are operated with ZVT and all diodes are turned off naturally.

Comparing the power consumption of the proposed driving circuits with that of the simplest driver shown in Fig. 2, we can find the effect of the energy recovery. Supplying the same dc-bus voltage, $200 \mathrm{~V}$, the average input current of the proposed circuit is $0.10 \mathrm{~A}$ and that of the simplest driver is $0.14 \mathrm{~A}$. Therefore, about $30 \%$ energy is recovered by the proposed circuit.

\section{CONCLUSION}

A regenerative driver for the PDP has been proposed in this paper. It utilizes inductors to resonate with the equivalent intrinsic capacitance of the PDP to avoid the abrupt charging/discharging operation. It not only achieves the energy recovery function, but also performs the soft-switching operation. All active switches are operated with zero-voltage switching and all diodes are turned off naturally without the problem of reverse recovery. Compared with prior approaches, the proposed sustain driving circuit is quite simple. The left-side and right-side circuits can be operated independently so it is not restricted to symmetrical operation and is suitable for practical applications. The presented circuit has been experimentally verified to be applicable on an 8-in PDP.

\section{REFERENCES}

[1] L. F. Weber, "Measurement of wall charge and capacitance variation for a single cell in AC plasma display panel," IEEE Trans. Electron Devices, vol. 24, pp. 533-538, Nov. 1997.

[2] C. L. Chen, S. T. Lo, C. C. Liu, and H. B. Hsu, "Study on the secondorder sustaining driver for plasma display panel," in Proc. 5th Asian Symp.Information Display, Hsinchu, Taiwan, R.O.C., Mar. 17-19, 1999, pp. 141-145.

[3] L. F. Weber and K. W. Warren, "Power efficient sustain drivers and address drivers for plasma panel," U.S. Patent 4866 349, Sept. 1989.

[4] T. Shinoda, "Method and a circuit for gradationally driving a flat display device," U.S. Patent 5541 618, July 1996.

[5] M. Ohba and Y. Sano, "Energy recovery driver for a dot matrix AC plasma display panel with a parallel resonant circuit allowing power reduction," U.S. Patent 5670 974, Sept. 1997.

[6] L. F. Weber and M. B. Wood, "Energy recovery sustain circuit for the AC plasma display," in SID'87 Dig., 1987, pp. 92-95.

[7] A. Tanaka, "Low power driver circuit for AC plasma display panel," U.S. Patent 5438 290, Aug. 1995.

[8] S. Y. Lin, C. L. Chen, C. C. Liu, and K. Lee, "Lossless sustain driver for plasma display panel," in Proc. Power Conversion and Intelligent Motion, vol. 38, Nuremberg, Germany, May 26-28, 1998, pp. 675-679.

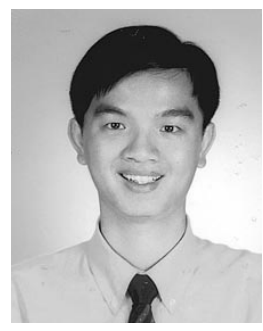

Horng-Bin Hsu was born in Taipei, Taiwan, R.O.C., in 1972. He received the B.S. degree in electrical engineering in 1997 from National Taiwan University, Taipei, Taiwan, R.O.C., where he is currently working toward the Ph.D. degree.

His current research is focused on the design of the driving circuit and driving waveform for plasma display panels.

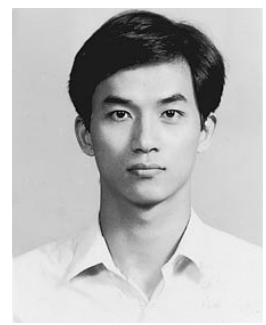

Chern-Lin Chen (S'86-M'90-SM'99) was born in Taipei, Taiwan, R.O.C., in 1962. He received the B.S. and $\mathrm{Ph} . \mathrm{D}$. degrees in electrical engineering from $\mathrm{Na}$ tional Taiwan University, Taipei, Taiwan, R.O.C., in 1984 and 1987, respectively.

Since 1987, he has been with the Department of Electrical Engineering, National Taiwan University, where he is currently a Professor. His current research interests lie in the areas of analysis, design, and application of power electronics converters and the control circuitry for plasma display panels.

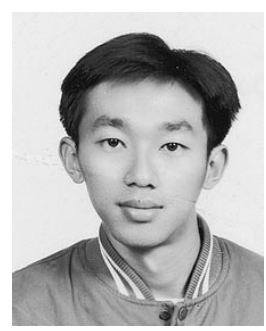

Song-Yi Lin was born in Nanto, Taiwan, R.O.C., in 1973. He received the B.S. and Ph.D. degrees in electrical engineering from National Taiwan University, Taipei, Taiwan, R.O.C., in 1994 and 1998, respectively.

Since 1998, he has been with Phoenixtec, Taipei, Taiwan, R.O.C., a company specializing in UPSs. 


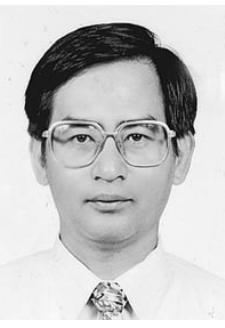

Kun-Ming Lee was born in Taiwan, R.O.C., in 1952.

$\mathrm{He}$ received the B.S. and M.S. degrees in electrical engineering from National Cheng-Kung University, Tainan, Taiwan, R.O.C., in 1974 and 1976, respectively. He is currently working toward the Ph.D. degree at National Taiwan University, Taipei, Taiwan, R.O.C.

He has been with the Acer Group since 1978 and is currently the Vice President of Acer Display Technology, Inc., Hsinchu, Taiwan, R.O.C. His current research is focused on circuit and system design. 